\title{
Iniciación a la danza como agente educativo de la expresión corporal en la educación física actual.Aspectos metodológicos \\ Initation to dance as an educational agent of body expression in the current physical education. Methodological aspects
}

\author{
Inmaculada García Sánchez, Raquel Pérez Ordás, África Calvo Lluch* \\ Universidad Pablo de Olavide de Sevilla
}

Resumen: La danza como manifestación rítmico-expresiva presenta amplias posibilidades para la formación del alumnado desde un punto de vista integrador (plano físico, intelectual y emocional). Sin embargo, la utilización de este contenido en el contexto de la educación física actual sigue siendo escaso y puntual. El desconocimiento de las aportaciones de la danza al desarrollo integral de la persona, la consideración eminentemente femenina de esta actividad y/o la falta de formación del profesorado, son algunas de las razones que podrían justificar el desaprovechamiento de la danza como agente educativo de la expresión corporal. Con respecto a la falta de formación del profesorado, este trabajo tiene el propósito de responder a una serie de interrogantes relacionados con el cómo enseñar danzas: ¿qué métodos pedagógicos son los más adecuados? ¿cualquier tipo de danza es idónea para trabajar en el aula? ¿cómo utilizar correctamente los elementos básicos del ritmo en las diferentes propuestas danzadas?

Palabra clave: Educación Física. Expresión Corporal. Danza.

Abstract: Dance as a rhythmic-expressive content presents ample opportunities for the training of students from an inclusive point of view (level of physical, intellectual and emotional). However, the use of this content or treatment in the context of physical education is limited or ad hoc. The ignorance of the contributions of dance to develop the whole person, considering this activity mainly women and/or lack of teacher training are some of the reasons that might justify the waste of dance as an educational agent of body expression. With regard to the lack of teacher training, the work presented below is intended to answer a series of questions related to how to teach dance: what methodology is appropriate?, any kind of dance is suitable to work in the classroom?, how to properly use the basic elements of rhythm in the proposals danced?.

Key words: Physical Education. Body Expression. Dance.

\section{Introducción}

Son muchos los autores que coinciden en destacar las posibilidades educativas que derivan del tratamientode la danzacomouna manifestación expresiva que contribuye al desarrollo físico, intelectual y afectivoemocional del individuo (Fructuoso y Gómez, 2001; Fuentes, 2006; Laban, 1978; Leese y Packer, 1991; Ortí y Balaguer, 2001; Ossona, 1984; Padilla y Hermoso, 2003; Rizo, 1996). Danzar supone practicar un ejerciciofísico corporalmente global, exentode elementos competitivos y con un alto componente hedonista (Laban, 1978; Ossona, 1984). De esta forma, la danza contribuye al desarrollo físico de la persona e incide positivamente en su salud y calidad de vida (Fuentes, 2006; Fructuoso y Gómez, 2001).

Por un lado, contribuye al desarrollo físico de la persona a través de una serie de factores, tales como: la adquisición y el desarrollo de habilidades y destrezas básicas; la adquisición y el desarrollo de tareas motrices específicas; el desarrollo de la coordinación y de habilidades perceptivo-motoras y el conocimiento y control corporal (Fuentes, 2006). Por otro lado, incide positivamente en la salud y calidad de vida de la persona permitiendo el desarrollo y mejora de la condición física, favoreciendo la interacción e integración social, facilitando la liberación de tensiones, canalizando el estrés y ayudando en el desarrollo de la capacidad creativa (Fructuoso y Gómez, 2001).

Padilla y Hermoso (2003) afirman que la danza, además de permitir un trabajo para la educación del cuerpo y del movimiento, abre el camino hacia el desarrollo de la creatividad, la relación entre compañeros, el conocimiento de uno mismo, el conocimiento de otras culturas y el desarrollo de la capacidad expresiva. En un contexto educativo, estos mismos autores (Padilla y Hermoso, 2003) proponen un modelo de danza divergente donde la creatividad, el juego y la socialización predominen sobre el modelo (la técnica), la repetición y la memorización de patrones de movimiento.

\footnotetext{
Fecha recepción: 29/12/2010 - Fecha envío revisores: 15-01-11 - Fecha de aceptación: 06-06-11 Correspondencia: Inmaculada García Sánchez

Crtra. Utrera km 1

41013 (Sevilla)

E-mail: igarcia@upo.es
}

El desconocimiento de las aportaciones de la danza al desarrollo integral de la persona, la consideración eminentemente femenina de esta actividad y/o la falta de formación del profesorado con respecto al tratamiento de este contenido rítmico-expresivo, son algunas de las razones que podrían justificar el desaprovechamiento de la danza como agente educativo de la expresión corporal en la educación física actual (Herrera, 2000; Paulson, 1993; Torre et al., 2007; McCarthy, 1996). Con respecto a la falta de formación del profesorado, el presente artículo persigue orientar o servir de ayuda al profesional tratando de dar respuesta a una serie de interrogantes, tales como: ¿cualquier tipo de danza es idónea para trabajar en el aula con mi alumnado? ¿qué metodología es la más adecuada para enseñar danzas? ¿utilizo correctamente los elementos básicos del ritmo en mis propuestas danzadas?. En primer lugar, y para facilitar la comprensión de los siguientes apartados, se hace necesario delimitar conceptualmente los términos de «expresión corporal» y de «danza».

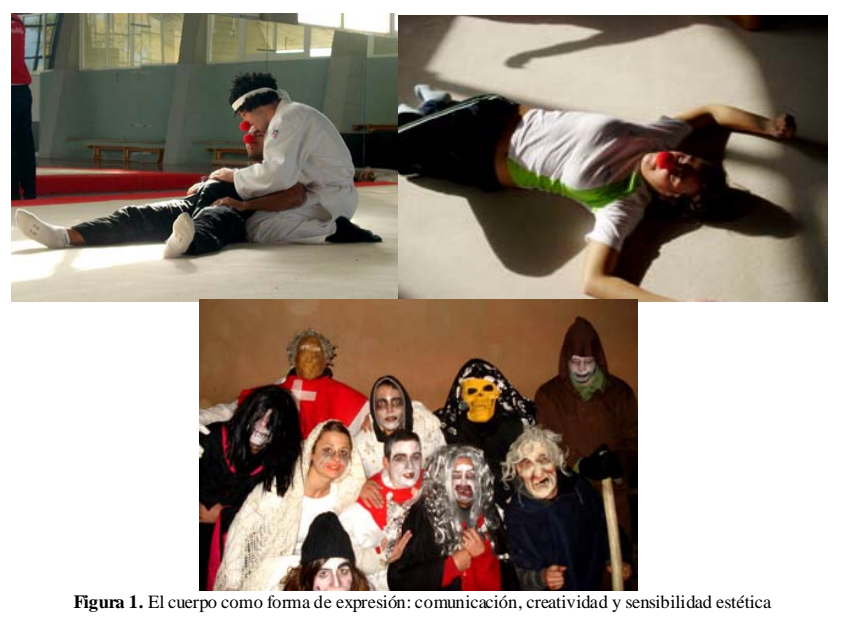

La expresión corporal es una disciplina que permite encontrar un lenguaje propio mediante el estudio y la profundización del empleo del cuerpo (Schinca, 2000). Esta definición parte del hecho de que todo ser humano, de unamanera consciente o inconsciente, se manifiesta mediante su cuerpo y utiliza su cuerpo como un instrumento irreemplazable de 
expresión que le permite ponerse en contacto con el medio y con los demás. El cuerpo se convierte en una forma de expresión que el individuo emplea en su comunicación habitual pero que puede aprender a utilizar mejor adquiriendo instrumentos que le permitan enriquecer su expresión, creatividad y sensibilidad estética (ver Figura 1).

La expresión corporal permite tomar conciencia del cuerpo y lograr su progresiva sensibilización utilizando el cuerpo plenamente, tanto desde el punto de vista motriz como desde su capacidad expresiva y creadora, para lograr la exteriorización de ideas y sentimientos (Stokoe y Schachter, 1994). De esta forma, el trabajo específico de expresión tiene cabida dentro de una serie de áreas que conciben la expresión corporal como una técnica con diferentes utilidades y finalidades (Mateu et al., 1999):

- Área psicológica: concibe la expresión corporal como una técnica al servicio del hombre con la que se consigue una mejor realización de sí mismo. En esta área, la expresión es un medio con finalidad terapéutica (p.e., la risoterapia, danzaterapia o abrazoterapia).

- Área artística: concibe la expresión como una técnica dramática con lenguaje propio (p.e., danza, mimo, pantomima o teatro). Desarrolla el perfeccionamiento de la técnica y enriquece el potencial expresivo que constituye el material de su actividad. En esta área, la expresión es un medio con finalidad profesional. El objetivo principal es la tecnificación para lograr un control preciso de toda la expresión corporal.

- Área metafísica: concibe la expresión como una supra-técnica en la que el componente corporal toma fuerza a través del componente mental. Su finalidad es la comunicación sobrenatural. En esta área, la expresión es un medio para el desarrollo espiritual.

- Área pedagógica: concibe la expresión como un procedimiento didáctico interdisciplinario. Se incorpora a la escuela como materia específica dentro de diferentes materias o asignaturas. En esta área, la expresión es un medio con finalidad educativa (Figura 2).

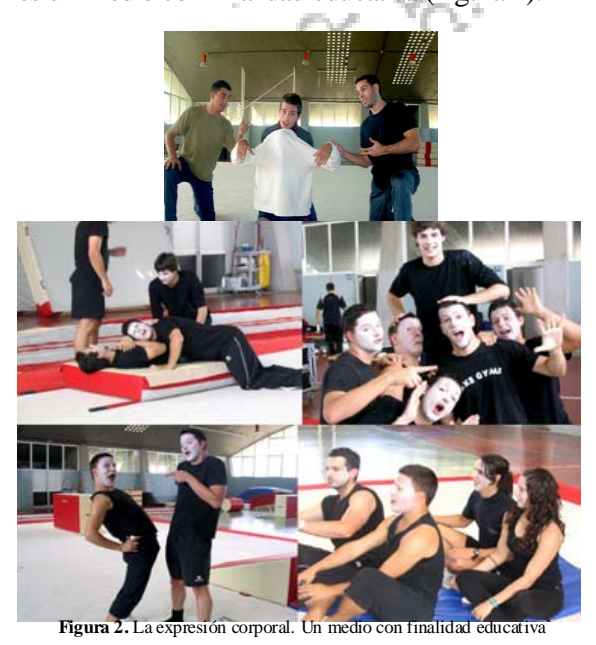

El término danza ha sido utilizado para designar diferentes conceptos e ideas siendo difícil la adopción de un único significado que describa con rigor la globalidad del término. La danza como expresión y comunicación no verbal, forma parte de un proceso social en el que diversos elementos, comportamientos y formas de comunicación, interaccionan en un contexto social determinado (Fuentes, 2006).

Numerosos autores han definido el concepto danza contemplando la generalidad y la particularidad del término desde una perspectiva integradora (Cuellar, 1996; Fuentes, 2006; García Ruso, 1997; Kraus, 1969; Ordás et al., 2010; Vicente et al., 2010). Así por ejemplo, Cuellar (1996) define danza como un lenguaje del cuerpo y a la vez una actividad motriz que combina armoniosamente en el espacio movimientos que una audición musical crea y ordena. Para Fuentes (2006), la danza es el movimiento que presenta unas características propias y que se articula en un espacio, tiempo y cadencia rítmica concretos. Junto a este componente motriz, la danza es expresión, comunicación o representación, que se basa en la gestualidad corporal y otros elementos secundarios como pueden ser las características de los bailarines (edad, sexo, físico...), el entorno visual (escenario, vestuario, iluminación, objetos...) y los elementos sonoros (música, ruidos y sonidos). Finalmente, Vicente et al. (2010) proponen un concepto de danza como interrelación de una serie de elementos que se condicionan y desarrollan mutuamente. Estos autores (Vicente et al., 2010) delimitan conceptualmente el término danza como la unidad formada por dos elementos esenciales, el motriz y el expresivo, que interactúan con unos elementos biológicos, psicológicos, sociales, culturales y estéticos determinados y se conforman a través de elementos espaciales, temporales y rítmicos.

2. Características del proceso de enseñanza-aprendizaje de cualquier tipo de danza

En un contexto educativo-recreativo, son muchos los autores que coinciden en afirmar que el proceso de enseñanza destinado al aprendizaje de cualquier tipo de danza o baile se caracteriza por tres aspectos: (1) la sencillez; (2) una metodología de enseñanza expresivo-vivencial y (3) la utilización del juego como elemento motivador y facilitador del aprendizaje (Arteaga et al., 1999; Castañer, 2000; Learreta et al., 2007; Montesinos, 2004; Montávez y Zea, 1998 y 2004; Ordás et al., 2010; Viciana y Arteaga, 2004) (figura 3):

- La sencillez en el proceso de enseñanza-aprendizaje de cualquier tipo de danza o baile, se refiere tanto a los movimientos que constituyen la danza (pasos de baile) como a la organización y evolución del grupo.

- Una metodología de enseñanza expresivo-vivencial es aquella que permite al alumnado utilizar sus propios recursos expresivos, desarrollar su capacidad creativa, implicarse en su proceso de aprendizaje y socializarse en el aula.

- Cuando se baila (al igual que cuando se realiza cualquier otra actividad motriz), cuerpo, espacio y tiempo se convierten en elementos que interactúan entre sí simultáneamente. De esta forma, para que el juego se convierta en un elemento motivador y facilitador del aprendizaje, las propuestas danzadas tienen que permitir al alumnado jugar con su cuerpo, con el espacio de baile, con la música y con los compañeros/as.

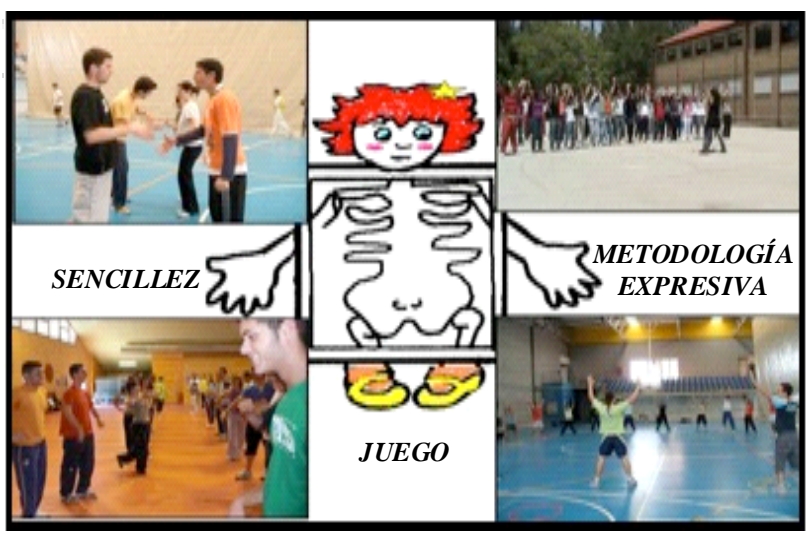

Figura 3. Características del proceso de enseñanza-aprend izaje de cualquier tipo de danza

3. La danza como interpretación intencional de un ritmo o de una música

Entre el cuerpo y la música se establece un elemento común: el movimiento; una medida común: el tiempo y una forma común de expresión: el ritmo. El elemento rítmico es el resultado de la capacidad de organizar los diferentes materiales sonoros en función de su intensidad dentro del factor tiempo (Castañer, 1988). El ritmo permite ordenar el cuerpo en el tiempo y en el espacio de una forma simultánea.

La danza es una de las actividades que desarrolla de forma especial el sentido rítmico y se puede entender como la interpretación intencional de un ritmo o de una música (Ortiz, 2008). De esta forma, para trabajar con la formación de estructuras coreográficas adaptadas a una música, los profesionales de cualquier ámbito de la actividad física y del deporte deben conocer y aprender a utilizar correctamente los elementos básicos del ritmo: 


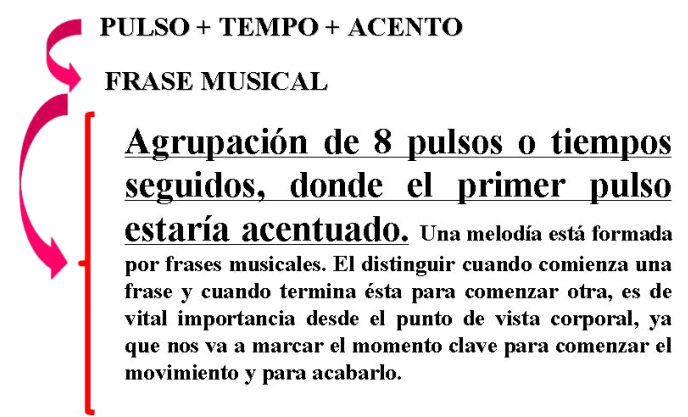

Figura 4. La frase musical

- Pulsos: son los tiempos regulares sobre los que cobra vida el ritmo. El ritmo base de una melodía corresponde a la sucesión continua e ininterrumpida de pulsaciones.

- Acentos: son las pulsaciones que destacan en intensidad y se repiten de forma periódica dentro del conjunto de pulsaciones.

- Tempo: es la frecuencia media del pulso musical (número de pulsaciones de una melodía en un minuto). Este elemento rítmico determina la velocidad a la que deben ejecutarse los movimientos que conforman cualquier forma danzada o baile.

- Frase musical: deriva de la utilización de los tres anteriores. Se trata de un elemento rítmico fundamental para la construcción de coreografías que destaca por su utilidad desde un punto de vista corporal (ver figura 4). Se trata de la sucesión de ocho tiempos o pulsos donde el primer pulso estaría acentuado.

- Compás: es la métrica del ritmo. Se trata del elemento rítmico más específico y menos necesario para trabajar el contenido danza en un contexto educativo. No obstante, es recomendable utilizar músicas con métricas sencillas (compases binarios y cuaternarios) que permitan al alumnado identificar el ritmo base de la música (los pulsos) de una forma fácil y rápida.

En danza, la música es fuerza motivadora que potencia la sensibilidad hacia el ritmo y la expresión corporal, genera el placer de ejecución del movimiento y provoca la atención por la estética del mismo. Se puede afirmar que la música es un instrumento pedagógico importante en el tratamiento de la motricidad y ofrece un buen repertorio de valores didácticos. No puede reducirse a la sola función de soporte rítmico del movimiento, sino que hay que saber explotar toda su riqueza si es que se la considera forma de complementación y organización del medio educativo. La presencia de la música debería de considerarse como una forma de organización del medio sonoro, que se introduce como variable que permite jugar con una gama mayor de factores que se desprenden tanto de la riqueza intrínseca de la música como del repertorio musical empleado (Pont, 1996).

\section{Una propuesta metodológica a través de las danzas de presentación}

Las danzas de presentación o animación persiguen trabajar la cohesión de grupo permitiendo una relación positiva entre los miembros (Figura 5). Dicho de otro modo, la ejecución de la danza tiene por objeto que el grupo se conozca, se relacione y se comunique. Además, la simplicidad en cuanto a los pasos de baile y a la organización-evolución del grupo (precisamente para que pueda darse esa comunicación e interacción entre los miembros) hace que cualquier persona, con unas nociones rítmicas básicas, pueda trabajar con este tipo de danzas (Viciana y Arteaga, 2004)

En las clases de educación física las danzas de presentación permiten iniciar al alumnado en el trabajo de danza como contenido rítmicoexpresivo de una forma sencilla, socializadora y lúdica. Inciden positivamente en el trabajo de socialización y cohesión de grupo e implican al alumnado en su proceso de enseñanza-aprendizaje desarrollando su capacidad creativa. Para facilitar la intervención didáctica

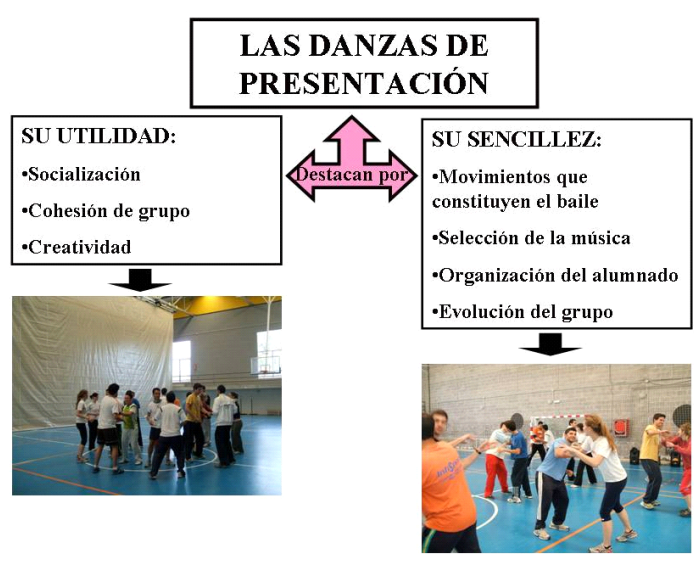

Figura 5. Características de las danzas de presentación

Figura 5. Ca

del docente en el aula, se presentan a continuación una serie de consideraciones metodológicas relacionadas con la selección de la música, la elaboración de los pasos de baile, la organización-evolución del grupo y el desarrollo de la capacidad creativa del alumnado.

\subsection{Selección de la música}

En este apartado se van a tener en cuenta dos criterios relacionados con la selección de la música:

a. Criterio rítmico:

- Pulsos y acentos bien marcados que permitirán discriminar con facilidad las frases musicales.

- En cuanto al tempo, es conveniente que no sea ni demasiado lento, ni demasiado rápido (p.e., entre 130 y 150 ppm). Trabajar con tempos muy rápidos, podría aumentar en exceso la intensidad del trabajo físico y/o la dificultad en la ejecución de los pasos de baile. Por el contrario, tempos demasiados lentos, podrían resultar desmotivantes y dificultarían igualmente la ejecución de los pasos de baile.

0. - Coreografiar entre cuatro y ocho frases musicales (según el nivel del grupo). Tener presente que cuanta más larga sea la coreografía, más pasos tendrá que memorizar el alumnado y mayor dificultad en el aprendizaje.

b. Criterio motivacional:

-La músicaha de cumplir elobjetivode activar yanimar al movimiento. Para ello, resultará muy interesante utilizar música adecuada a las edades del grupo con el que se trabaja.

- Para que las dinámicas tengan una continuidad en el tiempo y mantengan el nivel de activación del grupo, será conveniente utilizar música recopilada en pista única.

\subsection{Elaboración de los pasos de baile}

En este apartado se van a tener en cuenta dos aspectos relacionados con la elaboración de los pasos de baile:

a.Tipo de movimientos: los movimientos o pasos que constituyen el baile tendrán que ser sencillos. A modo de ejemplo, se podrán utilizar juegos de palmas, habilidades motrices básicas (p.e., giros, saltos, desplazamientos hacia delante o hacia atrás), diferentes formas de saludos o pasos básicos de aeróbic (p.e., paso en «V»).

b.Ejecución de movimientos:

-No enseñar más de dos pasos de baile por frase musical.

-La pareja ejecutará los pasos de baile de forma simultánea.

-La pareja ejecutará los pasos de baile con idéntica lateralidad.

\subsection{Organización y evolución del grupo (ver Figura 6)}

Con respecto a la organización y evolución del grupo, este apartado va a quedar estructurado en dos partes bien diferenciadas:

a.Organización inicial: parejas enfrentadas

-Las parejas enfrentadas se colocarán ubicadas libremente por el espacio.

-La pareja que enseñará la danza (p.e., el profesor/a con un alumno/ a) se situará en una posición destacada fuera del grupo. 
b.Tareas danzadas:

Tarea 1: «cuento y aprendo»

-Durante el proceso de enseñanza-aprendizaje de la danza, el alumnado contará en voz alta los ocho tiempos que dura cada frase musical. El conteo permitirá al alumnado conectar ritmo musical con ritmo corporal.

-Las parejas aprenderán la danza sin música y con una estrategia en la práctica analítica: paso/s de la primera frase musical (1); paso/s de la segunda frase musical (2); $1+2 ;$ paso/s de la tercera frase musical (3); 1 $+2+3$; paso/s de la cuarta frase musical (4); $1+2+3+4$; etc.

Tarea 2: «cambio de pareja»

-Las parejas bailarán al ritmo de la música con cambios de pareja. Estos cambios se llevarán a cabo libremente por el espacio en el tiempo que dura una serie musical (agrupación de cuatro frases musicales).

-La dinámica se mantendrá hasta que todos/as los alumnos/as hayan bailado con todos/as los alumnos/as.

Tarea 3: «círculos concéntricos»

-Las parejas se organizarán en círculos concéntricos. Los alumnos/ as que están situados en el círculo de afuera bailarán con los alumnos/as que están situados en el círculo de adentro.

-Los alumnos/as colocados en el círculo de afuera serán los que se desplazarán un puesto hacia la derecha para cambiar de pareja aprovechando los pasos de baile de la última frase musical.

-Una vez completado el círculo, las parejas cambiarán de posición. Si están adentro pasarán afuera y viceversa.
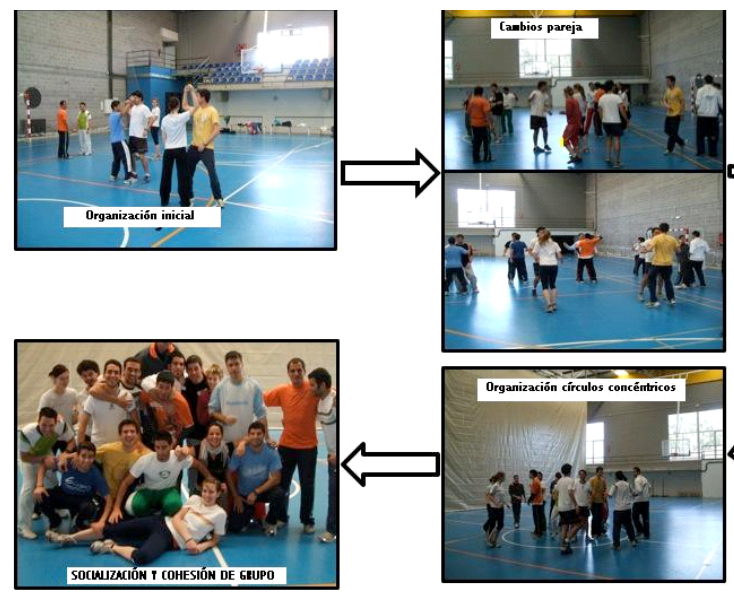

Figura 6. Danza de present ación: organización y evolución del grupø

\subsection{Desarrollo de la capacidad creativa}

Es muy importante destinar un espacio para el desarrollo de la capacidad creativa del alumnado. En esta propuesta danzada, cada pareja podrá inventar su propia danza de presentación siguiendo las orientaciones que se indican a continuación:

-Cada pareja inventará una danza utilizando sus propios recursos expresivos.

-Las parejas montarán la coreografía en lo que dura una serie musical (cuatro frases musicales).

-Durante el tiempo de creación, la música estará sonando.

-El tiempo de creación dependerá de las características del alumnado: experiencia previa con el trabajo de danza, nivel de motivación del grupo, grado de autonomía, nivel de responsabilidad, etc. Para este tipo de danzas, será suficiente destinar entre 15 y 25 minutos.

-El profesor/a irá evolucionando por el espacio para guiar y solucionar los problemas que vayan surgiendo en el proceso creativo de las parejas.

-Pasado el tiempo de creación, las parejas mostrarán su trabajo al resto de compañeros/as.

\section{Conclusiones}

Para valorar la danza como agente educativo de la expresión corporal es necesario que el educador reconozca que este contenido permite un trabajo para la educación del cuerpo y del movimiento, permite el desarrollo de la creatividad, la relación entre compañeros, el conocimiento de uno mismo, el conocimiento de otras culturas y el desarrollo de la capacidad expresiva del alumnado. En un contexto educativo-recreativo, el proceso de enseñanza-aprendizaje de cualquier tipo de danza o baile tiene que ser sencillo y lúdico. Para ello, es conveniente que el profesorado apueste por la utilización de una metodología de enseñanza expresivovivencial. Es importante destacar que para trabajar con la formación de estructuras coreográficas adaptadas a una música, los profesionales de cualquier ámbito de la actividad física y del deporte tienen que conocer qué son y para qué sirven los elementos básicos del ritmo.

Desde una perspectiva metodológica, la danza de presentación/ animación es una propuesta sencilla y accessible que incide positivamente en el trabajo de socialización y cohesión de grupo. La simplicidad en cuanto a los pasos que constituyen el baile y a la organización-evolución del grupo hacen posible que cualquier docente, con unas nociones rítmicas básicas, pueda utilizar este tipo de danzas en sus clases de educación física.

\section{Bibliografía}

Arteaga, M., Viciana, V.yConde, J. (1999). Desarrollo de la expresión corporal. Barcelona: Inde.

Castañer, M. (1988). El soporte musical en la gimnasia rítmica deportiva. Apunts. Educación Física y Deportes, 11-12, 37-41.

Castañer, M. (2000). Expresión Corporal y Danza. Barcelona: Inde.

Cuellar, M.J. (1996). Danza, la gran desconocida: Actividad Física paralela al Deporte. Boletim da Sociedade Portuguesa de Educaçao Física, 13, 89-98.

Fructuoso C., y Gómez, C. (2001). La danza como elemento educativo en el adolescente. Apunts. Educación Física y Deportes, 66, 31-37.

Fuentes, A.L. (2006). El valor pedagógico de la danza. Valencia: Universidad de Valencia, Servicio de Publicaciones.

García Ruso, H. Mª (1997). La danza en la escuela. Barcelona: Inde.

Herrera, S. (2000). Ver la música, escuchar el movimiento. LEEME (Revista Electrónica Europea de Música en la Educación), 5, 1-4.

Kraus, R. (1969). History of the dance in Art and Education. New Jersey: Prentice-Hall.

Laban, R. (1978). Danza educativa moderna. Buenos Aires: Paidós.

Learreta, B.; Ruano, K. y Sierra, M. A. (2007). Didáctica de la expresión corporal. Talleres monográficos. Madrid: Inde.

Leese, S. yPacker, M. (1991).Manual de danza. La danza en las escuelas, como enseñarlas y aprenderlas. Madrid: EDAF.

Mateu, M.; Durán, C. y Troguet, M. (1999). 1000 Ejercicios y juegos aplicados a las actividades de expresión. Barcelona: Paidotribo.

McCarthy, M. (1996). Dance in the music curriculum. Music Educators Journal, 82 (6), $17-$ 21.

Montávez, M., y Zea, M.J. (1998). Expresión Corporal. Propuestas para la acción. Córdoba: M.M y M.J.Z.

Montávez, M. yZea, M. J. (2004). Recreación Expresiva. Vol. Iy II. Málaga: recrea yEduca.

Montesinos, D. (2004). La expresión corporal. Su enseñanza por el método natural evolutivo. Barcelona: Inde.

Ordás, R., García, I., y Calvo A. (2010). Me muevo con la Expresión Corporal. Sevilla: MAD.

Ortí, J., y Balaguer, J. (2001). Dancemos al ritmo del «Hip-Hop». Apunts. Educación Física y Deportes, 65, 88-97.

Ortiz, M. (2008). La danza libre y la composición coreográfica en el ámbito educativo. En S. Galo, J. Coterón, J. Gil y A. Sánchez (eds.). El movimiento Expresivo (pp. 211218). Salamanca: Amarú ediciones.

Ossona, P. (1984). La educación por la danza. Barcelona: Paidós.

Padilla, C., y Hermoso Y. (2003). «Siglo XXI: perspectivas de la danza en la escuela. Tavira: revista de Ciencias de la Educación, 18, 9-20.

Paulson, P. (1993). New work in dance education. Arts Education Policy Review, 95 (1), 31-35

Pont, P. (1996). El material y la música como recursos en un programa de actividades físicas dirigido a personas mayores. Apunts. Educación Física y Deportes, 43, 7588.

Rizo, G (1996). La enseñanza de los bailes y las danzas tradicionales en la escuela: un enfoque interdisciplinar. Eufonía. Didáctica de la música, 3, 73-83.

Schinca, M. (2000). Expresión corporal. Técnica y expresión del movimiento. Barcelona: Praxis.

Stokoe, P. y Schachter, A. (1994). La expresión corporal. Barcelona: Paidós.

Torre, E., Palomares, J., Castellano, R. y Pérez, D.M. (2007). LA Expresión Corporal en el currículo educativo de la República de Cuba: Estudio de las necesidades en la formación inicial. Retos. Nuevas tendencias en Educación Física, Deporte y Recreación, 11, 11-16.

Viciana, V., y Arteaga, M. (2004). Las Actividades Coreográficas en la Escuela. Danzas, Bailes, Funky, Gimnasia-Jazz. Barcelona: Inde.

Vicente, G., Ureña, N., Gómez, M., y Carrillo, J. (2010). La danza en el ámbito educativo. Retos. Nuevas tendencias en Educación Fúsica, Deporte y Recreación, 17, 42-45. 